\title{
Validation of the Polish version of the Critical Care Pain Observation Tool (CPOT) to assess pain intensity in adult, intubated intensive care unit patients: the POL-CPOT study
}

Katarzyna Kotfis ${ }^{1}$, Małgorzata Zegan-Barańska ${ }^{1}$, Marta Strzelbicka ${ }^{1}$, Krzysztof Safranow ${ }^{2}$, Maciej Żukowski ${ }^{1}$, E. Wesley Ely³; the POL-CPOT Study Group

\author{
'Department of Anaesthesiology, Intensive Therapy and Acute Intoxications, \\ Pomeranian Medical University, Szczecin, Poland \\ ${ }^{2}$ Department of Biochemistry and Medical Chemistry, Pomeranian Medical University, \\ Szczecin, Poland \\ ${ }^{3}$ Vanderbilt University School of Medicine, Medicine/Allergy, Pulmonary, and Critical \\ Care, Veterans Affairs Geriatric Research Education Clinical Center (GRECC) \\ for Tennessee Valley, Nashville, Tennessee, USA
}

Submitted: 22 May 2017

Accepted: 1 August 2017

Arch Med Sci 2018; 14, 4: 880-889

DOI: https://doi.org/10.5114/aoms.2017.69752

Copyright (c) 2017 Termedia \& Banach

\begin{abstract}
Introduction: Pain in the critically ill affects nearly $50 \%$ of patients. In patients unable to self-report pain, behavioural scales are used. The aim of this study was to validate the Polish version of the Critical Care Pain Observation Tool (CPOT).

Material and methods: The prospective observational cohort study included patients observed during non-nociceptive and nociceptive procedures, at rest, during the intervention, and 15 min after each intervention. Assessments included self-report by patients and CPOT assessment carried out by two blinded observers.

Results: A total of 71 patients were included in the study (mean age: 66 years), predominantly males (50/71, 70\%), mean APACHE II score 26.04 \pm 10.56 . Results showed an excellent inter-rater correlation (ICC) between raters (ICC scores >0.97). Self-report NRS (numeric rating scale) scores were available from 58/71 patients (82\%). Patients' self-reported pain and CPOT showed a very strong correlation (Spearman's $R>0.85, p<0.0001$ ). The CPOT has high diagnostic value for detection of presence of patients' self-reported pain (ROC AUC $=0.938$ for rater $A$ and 0.951 for rater $B, p<0.0001$ ). CPOT score $\geq 2$ is an optimal cut-off to detect pain during a nociceptive procedure. A significantly higher mean CPOT score during a nociceptive procedure as compared to a non-nociceptive procedure or at rest was found $(p<0.0001)$. Conclusions: This study shows that the Polish version of the CPOT can be used to assess pain in critically ill patients with no hypnotic, opioid-based analgo-sedation. Polish CPOT scores correlated well with patients' self-reported presence of pain and showed excellent inter-rater reliability. This makes the Polish version of the CPOT a reliable pain assessment tool.
\end{abstract}

Key words: pain, intensive care, pain intensity, Critical Care Pain Observation Tool, pain measurement tool.

\section{Introduction}

Critically ill patients frequently experience pain during their stay in the intensive care unit (ICU). Nearly $30 \%$ of ICU patients suffer from pain

\author{
Corresponding author: \\ Katarzyna Kotfis MD, PhD, \\ DESA \\ Department \\ of Anaesthesiology, \\ Intensive Therapy and \\ Acute Intoxications \\ Pomeranian Medical \\ University \\ Teaching Hospital No. 2 \\ 70-111 Szczecin \\ E-mail: katarzyna.kotfis@ \\ pum.edu.pl
}


at rest and up to $50 \%$ suffer pain during nursing procedures [1]. Acute pain experienced in the ICU may become a chronic problem after discharge from the unit, as a lifelong ICU footprint. Everyday nursing procedures and interventions (i.e. positioning, mouth and trachea suctioning, wound care, catheter removal or placement, cannulation or intubation) may be a potential source of pain; therefore there is a clinical need for a simple and easy ICU pain scale available in every language to evaluate this condition in patients unable to self-report pain.

Assessment of pain in patients treated in the ICU is a daily clinical challenge. Various guidelines regarding assessment of pain, agitation and delirium in critically ill patients (PAD guidelines from the year 2013, DAS guidelines from 2015) and recommendations (eCASH from 2016) exist to guide the ICU care team in the process of pain evaluation and management [2-4]. The area of pain monitoring is traditionally the weakest part of the PAD Guidelines in terms of data on the tools. That is because this particular element of suffering is very subjective and the resultant intra-class correlation coefficient (ICC) is generally lower than barometers of reliability and validity for agitation/ arousal and delirium instruments [2].

The gold standard for pain assessment is the patient's self-report of pain using the Numeric Rating Scale (NRS) or Visual Analog Scale (VAS), which can be aided with behavioural scales only when the self-report is impossible to obtain. Changes in vital signs (heart rate, blood pressure or respiratory rate) are not reliable measures for pain as their fluctuations may either be associated with the underlying pathology or may represent the side effects of suffering. The observational pain scales include the Critical-Care Pain Observation Tool (CPOT) and the Behavioural Pain Scale (BPS) and have been recommended and validated for clinical use in critically ill adults $[5,6]$. However, their validation in a given patient population (cardiac, burn, different languages) is strongly recommended and required. It has been shown that implementation of systematic pain assessment, including evaluation by the medical team (doctors, nurses, physiotherapists) as well as family members, to identify pain-related behaviour aids better identification of patients' needs [5].

It is clear therefore why effective pain management has been identified as one of the first priorities in a humanized ICU approach, but there is still room for improvement in this area. The above-mentioned pain scales have not been officially translated into Polish or validated in Poland. The need to equip critical care teams with dedicated monitoring tools is clear, as is the understanding that early identification of pain warrants early and adequate treatment. The CPOT has been developed by ICU professionals, yet it has not been translated or validated in Polish until now. The aim of this study was to validate the CPOT translated into Polish using inter-rater reliability, discriminant validity and criterion validity.

\section{Material and methods}

\section{CPOT translation}

For the purpose of this study and further use, the CPOT was translated from English into Polish and received a back-translation after written permission was obtained from the author (C. Gelinas). The Polish CPOT translation was published and is available for general use [7]. Next, official permission was granted by the American Association of Critical Care Nurses to use the translated scale. This step was followed by a period of intensive theoretical and bedside training regarding the CPOT that led to this study.

The Polish version of the CPOT is a direct translation of the English version. To verify the linguistic accuracy of the translation it was back-translated and evaluated by an English-speaking research team from Vanderbilt University on behalf of the first author of the original study. The CPOT includes four behavioural domains and was designed to specifically detect pain in critically ill adults. The four categories are: facial expressions, body movements, muscle tension and either compliance with the ventilator (for patients who are intubated) or verbalisation (for patients who are extubated). In each category a score of 0,1 or 2 can be assigned to a total of between 0 and 8 points. There are no specific differences in the Polish version as compared to the English version of the CPOT.

\section{Study population}

A prospective observational cohort study was performed including 71 eligible intubated adult patients at the 17-bed Intensive Care Unit of the Department of Anaesthesiology, Intensive Therapy and Acute Intoxications of the tertiary teaching hospital (Teaching Hospital No. 2) of the Pomeranian Medical University in Szczecin, Poland between December 2016 and March 2017. The unit has implemented a no-hypnotic analgesia-based protocol (fentanyl and morphine intravenous infusion) for intubated patients. The opioid-based analgosedation regime was aided with non-opioid analgesics (metamizole, paracetamol) and adjuvants (lignocaine) as this has been shown to act as an opioid-sparing approach.

\section{Ethical concerns}

This observational study was presented to the Bioethical Committee of the Pomeranian Medical 
University on 21.12.2016 and the requirement for written informed consent was waived because of the non-interventional character of this study. Pain was assessed during routine everyday nursing procedures such as changing patient position and eye wiping - both regarded as ICU daily care. Also patient self-reporting of pain was part of routine care by the bed-side nurses in our ICU. All patients admitted to the ICU were evaluated by two members of the study team based on the inclusion and exclusion criteria and re-evaluated by the main investigator.

\section{Inclusion criteria}

- Age above 18 years,

- Ability to communicate in the Polish language,

- Intubated or with tracheostomy for more than $48 \mathrm{~h}$ before inclusion with or without mechanical ventilation (controlled modes, spontaneous modes),

- Richmond Agitation Sedation Scale (RASS) score above or equal to -3 ,

- Unrestricted sight and hearing,

- No limitations for body position changing,

- With no important intervention within the last $48 \mathrm{~h}$ prior to inclusion in the study (i.e. operation, tracheostomy).

\section{Exclusion criteria}

- A medical need for deep sedation - treatment of severe respiratory failure associated with patient-ventilator dyssynchrony, preventing awareness during neuromuscular blockade, status epilepticus, certain surgical conditions requiring immobility, cases of severe brain injury with intracranial hypertension,

- Facial trauma (unable to evaluate facial expression),

- RASS -4 or -5 ,

- Neurological or psychiatric disorders,

- Use of neuromuscular blocking agents,

- Regular narcotic users,

- Chronic pain syndrome patients.

\section{Study measures}

Each assessment was performed by two members of the study team, who obtained both theoretical and practical training from the primary investigator (PI)/first author (KK). To provide multiple inter-rater variability opportunities the evaluation was performed by different healthcare providers (rater $A$ and rater $B$ ), rater $A$ always being the $\mathrm{PI}$ (physician, ICU consultant) and rater $\mathrm{B}$ being one of 3 ICU physicians (1 ICU consultant, 2 senior ICU residents), 4 senior ICU nurses or 1 ICU physiotherapist. The CPOT was evaluated concomitantly by two raters blinded to each other and patient assessment was performed once a day, during daytime hours. Pain intensity was evaluated during routine nursing procedures in the ICU, both non-nociceptive procedures (NNP) - eyelid wiping with normal saline - and nociceptive procedures (NP) - patients' positioning and turning.

The assessments were performed at fixed time points: $5 \mathrm{~min}$ prior to the procedure, during the procedure and $15 \mathrm{~min}$ after the procedure. Each assessment included NRS self-report by the patient (if possible) and CPOT assessment carried out by two observers blinded to each other. As two types of procedures were performed, a total of 6 assessments was obtained from each rater. Altogether the time points were as follows: series I - non-nociceptive - T1 ( 5 min before NNP), T2 (at NNP), T3 (15 min after NNP); and series II - nociceptive - T4 (5 min before NP), T5 (at NP), T6 (15 min after NP).

Basic demographic data collected include: sex, age, APACHE II (Acute Physiology and Chronic Health Evaluation II) score within $24 \mathrm{~h}$ of admission, admission source (internal medicine vs surgery), data regarding mechanical ventilation of intubated patients, type of mechanical ventilation - controlled modes (BIPAP, SIMV) or spontaneous modes (CPAP) - and use of co-analgesics during the $24 \mathrm{~h}$ prior to study inclusion. The level of consciousness and arousal was assessed using the Polish version of the Richmond Agitation Sedation Scale (RASS), ranging from -5 (no reaction to voice or touch) to +4 (combative), whereas target RASS was 0 (calm, cooperative patient). The ICU delirium screening was performed using the Polish version of Confusion Assessment Method for ICU (CAM-ICU). Both sedation and delirium assessment was performed prior to CPOT evaluation.

The patient's self-report was initiated by the bed-side nurse taking care of the patient with a question - Do you feel pain? - a NO answer was regarded as equivalent to NRS of 0 , whereas a YES answer led to NRS assessment using a pre-printed large scale for the patient to show the number from 0 (no pain) to 10 (the worst pain ever). The CPOT was developed as means of pain assessment in critically ill patients and covers 4 behavioural categories - facial expression, body movement, ventilator synchrony and muscle tone. In each category the raters can appoint from 0 to 2 points, up to a total amount of 0 to 8 points.

This prospective observational cohort study was registered at ClinicalTrials.gov website and received the following identifier: NCT03024528.

\section{Statistical analysis}

Baseline characteristics of the study group were analysed using descriptive statistics and were presented with either mean \pm SD for quantitative parameters and numbers and percentages for qual- 
itative parameters. To assess the psychometric properties of the CPOT, a methodology consistent with previously reported studies was applied [5, 8, 9]. Inter-rater reliability was calculated using intraclass correlation coefficients (ICC 3.1) for the СРОT. Based on previous studies the sample size required for validating CPOT established using precision ICC (3.1) was estimated to be between 55 and 65 patients (precision of ICC of $0.85 \pm 0.10$, for a scale with four subscales) [5]. In order to test the criterion validity of the CPOT we performed receiver operating characteristic (ROC) curve analysis and determined the cut-off value for optimal sensitivity and specificity for the detection of the patient's self-reported pain. Criterion validity was tested by Spearman's rank correlation coefficient (Rs) between CPOT and NRS scales. The Rs was also used to analyse correlations between CPOT scores and physiological indicators. We used the Wilcoxon signed-rank test for discriminant validity assessment by comparison of CPOT values between time points. Statistical significance was set at $p<0.05$. Statistical analysis was performed using Excel (Microsoft) and Statistica 13 with Medical Bundle 4.0 (StatSoft Inc.).

\section{Results}

\section{Baseline descriptive statistics}

To perform this prospective cohort study we screened 99 patients for eligibility, yet 28 were excluded (15 required deep sedation due to patient-ventilator asynchrony, 4 required an emergency operation or re-operation, 4 - chronic pain syndromes, 3 - psychiatric reasons, 1 - surgical immobility, 1 - deep sedation due to status epilepticus). A total of 71 patients were included in the study (mean age: $65.7 \pm 12.2$ ), predominantly males $(70.42 \%, 50 / 71)$ with a mean APACHE II score $24 \mathrm{~h}$ within admission to the ICU of 26.0 \pm 10.6 . The majority of the patients were admitted from a surgical unit $(61.97 \%, 44 / 71)$, on CPAP (70.42\%, 50/71) and screened positive for ICU delirium (CAM-ICU positive) - 53.52\% (38/71). None of the patients received a sedative agent at the time of assessment and 35.22\% (25/71) received no opioid infusion. Of the remaining $64.78 \%$ $(46 / 71)$ of patients receiving an opioid infusion, 49.29\% (35/71) received intravenous fentanyl infusion and $15.49 \%(11 / 71)$ received intravenous morphine infusion. The use of co-analgesics was recorded as follows: none $-45.07 \%$ (32/71); paracetamol - 26.76\% (19/71); metamizole (dipyrone) $-29.58 \%$ (21/71); and lignocaine $-4.23 \%$ (3/71). All the baseline data are depicted in Table I.

The distribution of the NRS for patients' self-reported pain (where available) and CPOT values for rater $A$ and rater $B$ at each assessment time (T1-T6) is shown in Table II.

\section{Inter-rater reliability for Polish CPOT}

The intraclass correlation coefficients (ICC) for rater $\mathrm{A}$ and rater $\mathrm{B}$ scored $>0.85$ at each measured time point (Table III), i.e. at rest (T1, T3, T4, $\mathrm{T} 6$ ), at the non-nociceptive procedure (T2) and at the nociceptive procedure (T5). High intraclass correlation coefficients confirmed inter-rater reliability of the Polish version of the CPOT.

\section{Criterion validity for Polish CPOT}

Data in Table IV show a very strong correlation between pain self-reported by the patient using the NRS at T1, T2 and T5 and pain assessed by both rater $\mathrm{A}$ and rater $\mathrm{B}$ using the CPOT $(R s>0.85$, $p<0.0001)$. The results of the NRS were available for $58 / 71$, meaning that $81.7 \%$ of patients were able to self-report pain using the NRS.

Table I. Baseline characteristics of study group

\begin{tabular}{|c|c|}
\hline Variable & Value $(n=71)$ \\
\hline Age, mean \pm SD [years] & $65.7 \pm 12.2$ \\
\hline \multicolumn{2}{|l|}{ Gender, $n(\%)$ : } \\
\hline Female & $21(29.58)$ \\
\hline Male & $50(70.42)$ \\
\hline $\begin{array}{l}\text { APACHE II score within } 24 \mathrm{~h} \text { of } \\
\text { admission, mean } \pm \text { SD }\end{array}$ & $26.0 \pm 10.6$ \\
\hline \multicolumn{2}{|l|}{ Admission to ICU, $n(\%)$ : } \\
\hline Surgical & $44(61.97)$ \\
\hline Medical & $27(38.03)$ \\
\hline \multicolumn{2}{|l|}{ Mode of ventilation, $n(\%)$ : } \\
\hline SIMV & $12(16.90)$ \\
\hline BIPAP & $9(12.68)$ \\
\hline CPAP & $50(70.42)$ \\
\hline \multicolumn{2}{|l|}{ Analgesia - opioids, $n$ (\%): } \\
\hline None & $25(35.22)$ \\
\hline Fentanyl & 35 (49.29) \\
\hline Morphine & $11(15.49)$ \\
\hline \multicolumn{2}{|c|}{ Co-analgesia - non-opioid analgesics, $n(\%)$ : } \\
\hline None & $32(45.07)$ \\
\hline Paracetamol & $19(26.76)$ \\
\hline Metamizole & $21(29.58)$ \\
\hline Lignocaine & $3(4.23)$ \\
\hline \multicolumn{2}{|l|}{ CAM-ICU, $n(\%):$} \\
\hline Positive - ICU delirium present & $38(53.52)$ \\
\hline Negative - ICU delirium absent & $33(46.48)$ \\
\hline
\end{tabular}

ICU - intensive care unit, CAM-ICU - Confusion Assessment Method for ICU, $n$-number of patients. 
Table II. Distribution of the values for patients' self-reported pain (NRS available in 58 patients), rater A and rater B CPOT assessment at each measured time

\begin{tabular}{|lcccc|}
\hline Assessment & Time & $\begin{array}{c}\text { NRS self-report } \\
\text { (mean } \pm \text { SD) }\end{array}$ & $\begin{array}{c}\text { CPOT rater A } \\
\text { (mean } \pm \text { SD) }\end{array}$ & $\begin{array}{c}\text { CPOT rater B } \\
\text { (mean } \pm \text { SD) }\end{array}$ \\
\hline Before NNP & T1 & $0.55 \pm 1.14$ & $0.76 \pm 1.26$ & $0.73 \pm 1.18$ \\
\hline NNP & T2 & $0.64 \pm 1.13$ & $0.83 \pm 1.26$ & $0.79 \pm 1.26$ \\
\hline After NNP & T3 & $0.52 \pm 0.98$ & $0.70 \pm 1.21$ & $0.70 \pm 1.25$ \\
\hline Before NP & T4 & $0.55 \pm 1.07$ & $0.70 \pm 1.22$ & $0.72 \pm 1.23$ \\
\hline NP & T5 & $2.84 \pm 1.98$ & $3.18 \pm 1.88$ & $3.15 \pm 1.93$ \\
\hline After NP & T6 & $0.74 \pm 1.13$ & $0.96 \pm 1.31$ & $0.92 \pm 1.27$ \\
\hline
\end{tabular}

NRS - Numeric Rating Scale, NNP - non-nociceptive procedure, NP-nociceptive procedure.

Table III. Distribution of ICC for rater A vs. rater B at each measured assessment

\begin{tabular}{|lcc|}
\hline Assessment & Time & ICC: rater A/rater B \\
\hline Before NNP & T1 & 0.972 \\
\hline NNP & T2 & 0.987 \\
\hline After NNP & T3 & 0.972 \\
\hline Before NP & T4 & 0.995 \\
\hline NP & T5 & 0.973 \\
\hline After NP & T6 & 0.979 \\
\hline
\end{tabular}

ICC - intra-class correlation coefficient, NNP - non-nociceptive procedure, NP-nociceptive procedure.

Figure 1 shows scatterplots depicting strong correlations between NRS results and CPOT evaluated by rater $\mathrm{A}$ and rater $\mathrm{B}$ at $\mathrm{T} 5$ (nociceptive procedure).

\section{The ROC for Polish version of CPOT}

The values for sensitivity and specificity of the Polish version of the CPOT for different cut-off values are shown in Table $\mathrm{V}$.

The area under the curve (AUC) values for the CPOT to detect pain for all given assessment times are shown in Table VI. No significant differences between raters $A$ and $B$ were found.

Both sensitivity and specificity for the Polish version of the CPOT are high for each study period - T1, T2 and T5 - for both rater A and rater B, as

A Scatterplot for correlation between T5B_CPOT and T5_NRS

$$
\begin{array}{r}
\text { POL-CPOT sta } 63 v^{*} 71 \mathrm{c} \\
\text { T5B }=0.6158+0.7957 x
\end{array}
$$

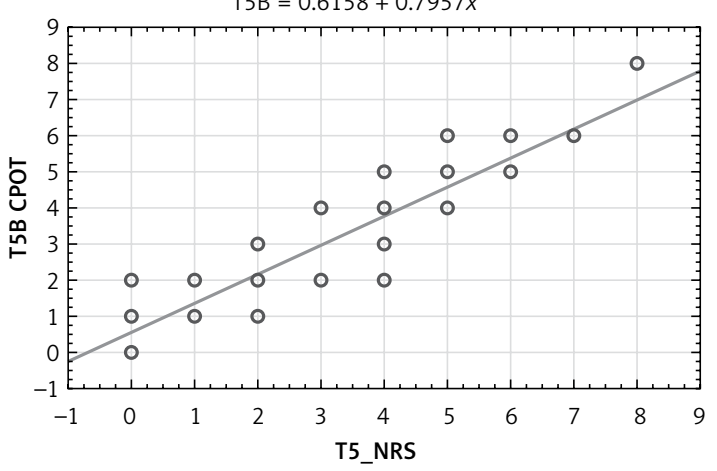

Table IV. Spearman rank correlation coefficients for correlations between Critical Care Pain Observation Tool (CPOT) and self-reported pain (NRS) values for 2 raters at 3 time points, $n=58$

\begin{tabular}{|c|c|c|c|}
\hline Assessment & Time & $\begin{array}{c}\text { NRS-CPOT } \\
\text { correlation } \\
\text { rater A }\end{array}$ & $\begin{array}{c}\text { NRS-CPOT } \\
\text { correlation } \\
\text { rater } \mathrm{B}\end{array}$ \\
\hline Before NNP & $\mathrm{T} 1$ & $0.882^{*}$ & $0.873^{*}$ \\
\hline NNP & $\mathrm{T} 2$ & $0.873^{*}$ & $0.852^{*}$ \\
\hline NP & T5 & $0.885^{\star}$ & $0.892^{*}$ \\
\hline
\end{tabular}

depicted in Figure 2. The receiver operating characteristic curves (ROC) at T1, T2 and T5 for both raters are shown in Figures 3-5.

The ROC curve at rest (Figure 3), at non-nociceptive procedure (Figure 4) and nociceptive procedure (Figure 5).

\section{Discriminant validity for Polish CPOT}

The discriminant validity for the Polish version of the CPOT was also high. Table VII shows CPOT ranges at specified observation times for rater $A$ and rater B. Both methods, NRS and CPOT, show higher scores during pain than at rest or during non-nociceptive procedures, as shown in Figures 6-8.

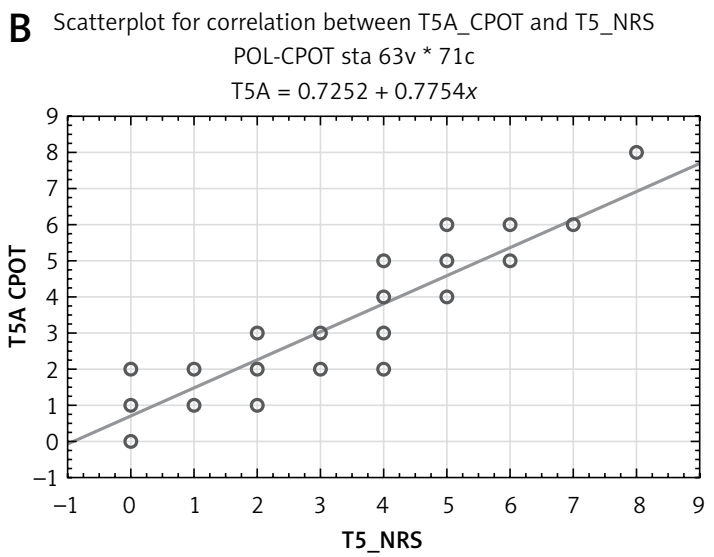

Figure 1. Scatterplot for correlation between NRS and CPOT reported at T5 by rater A (T5A) and rater B (T5B) 
Table V. Sensitivity and specificity for CPOT cut-off values to detect patients' self-reported pain at nociceptive procedure (T5)

\begin{tabular}{|lcccccc|}
\hline CPOT & $\begin{array}{c}\text { Sensitivity } \\
\text { rater A } \\
(\%)\end{array}$ & $\begin{array}{c}\text { Specificity } \\
\text { rater A } \\
(\%)\end{array}$ & $\begin{array}{c}\text { Correctly } \\
\text { classified } \\
\text { rater A (\%) }\end{array}$ & $\begin{array}{c}\text { Sensitivity } \\
\text { rater B } \\
(\%)\end{array}$ & $\begin{array}{c}\text { Specificity } \\
\text { rater B } \\
(\%)\end{array}$ & $\begin{array}{c}\text { Correctly } \\
\text { classified } \\
\text { rater B (\%) }\end{array}$ \\
\hline$\geq 1$ & 100 & 30 & 87.9 & 100 & 30 & 87.9 \\
\hline$\geq 2$ & 91.7 & 80 & 89.7 & 91.7 & 90 & 91.4 \\
\hline$\geq 3$ & 66.7 & 100 & 72.4 & 60.4 & 100 & 67.2 \\
\hline$\geq 4$ & 39.6 & 100 & 50 & 41.7 & 100 & 51.7 \\
\hline$\geq 5$ & 22.9 & 100 & 36.2 & 20.8 & 100 & 34.5 \\
\hline$\geq 6$ & 10.4 & 100 & 25.9 & 12.5 & 100 & 27.6 \\
\hline
\end{tabular}

CPOT - Critical Care Pain Observation Tool.

Table VI. ROC AUC for CPOT assessments performed by rater A and rater B to detect patients' self-reported pain

\begin{tabular}{|lcccc|}
\hline Assessment & Time & AUC Rater A & AUC Rater B & $P$-value \\
\hline Before NNP & T1 & 0.978 & 0.974 & 0.79 \\
\hline NNP & T2 & 0.950 & 0.926 & 0.48 \\
\hline After NNP & T3 & 0.933 & 0.948 & 0.18 \\
\hline Before NP & T4 & 0.912 & 0.916 & 0.53 \\
\hline NP & T5 & 0.938 & 0.951 & 0.89 \\
\hline After NP & T6 & 0.918 & 0.869 & 0.13 \\
\hline
\end{tabular}

AUC - area under the ROC curve, NNP - non-nociceptive procedure, NP-nociceptive procedure, $p$ - statistical significance for difference between AUC of rater $A$ and $B$.

A

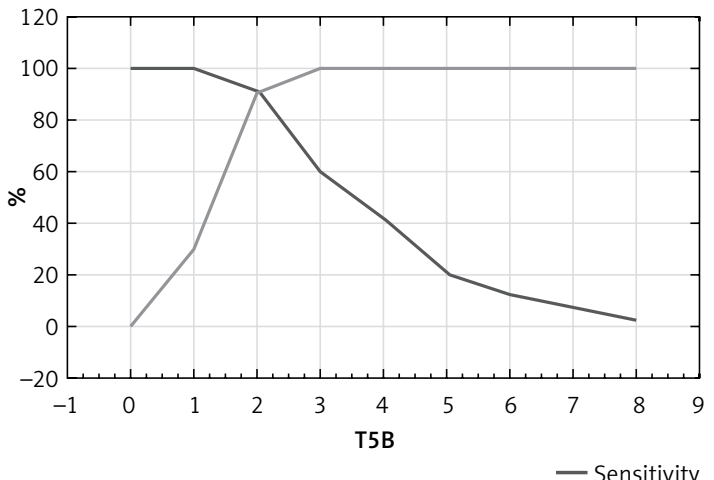

B

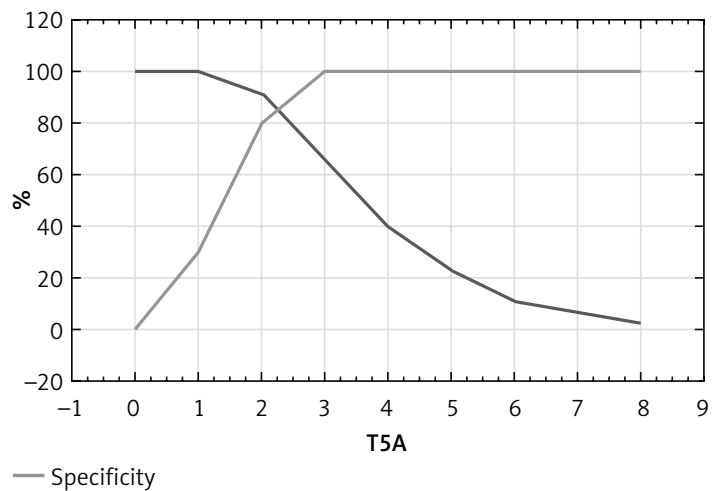

Figure 2. Sensitivity and specificity for CPOT at nociceptive procedure (T5) for rater $A(T 5 A)$ and rater $B(T 5 B)$ - the proposed cut-off value for CPOT is set at 2

Table VII and Figures 6-8 show that both methods, NRS and CPOT, show higher scores during nociceptive procedures (T5) than at rest (T4, T6) or during non-nociceptive procedures (T1-T3); the results are statistically significant.

\section{Correlation between CPOT and physiological parameters}

Table VIII shows Spearman's rank correlation coefficients for the CPOT and vital signs (HR, systolic, diastolic and mean arterial pressure). A negative correlation means that higher CPOT values correspond to lower vital signs. Statistical significance was found only for negative correlation of diastolic blood pressure and CPOT at rest (T1), at the non-nociceptive procedure (T2) and during the nociceptive procedure (T5), for both raters (A and B). No correlation was found between CPOT scores and physiological indicators, apart from diastolic blood pressure.

\section{Discussion}

The POL-CPOT study has validated the Polish version of the Critical Care Pain Observation Tool, introducing this assessment tool to ICUs 


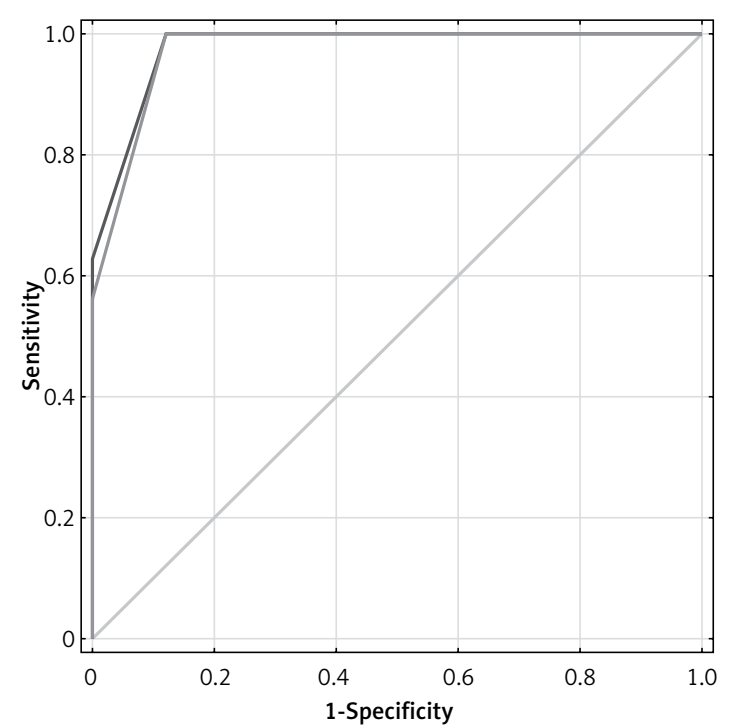

- T1A - T1B Reference line

Figure 3. Receiver operating characteristic curves $(\mathrm{ROC})$ at rest $(\mathrm{T} 1)$ for rater $\mathrm{A}(\mathrm{AUC}=0.978)$ and rater B (AUC $=0.974)$

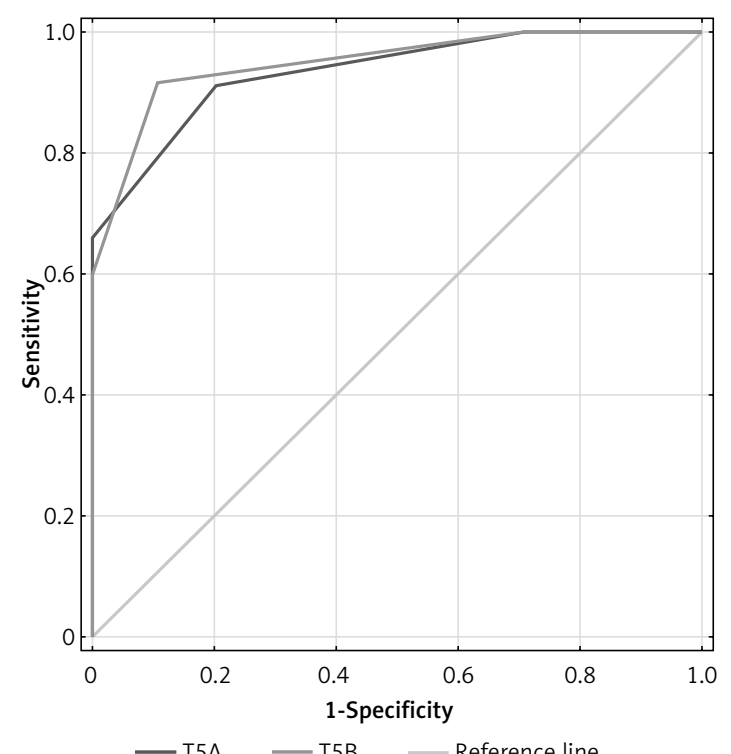

Figure 5. Receiver operating characteristic curves (ROC) during nociceptive procedure (T5) for rater $A(A \cup C=0.938)$ and rater $B(A \cup C=0.951)$

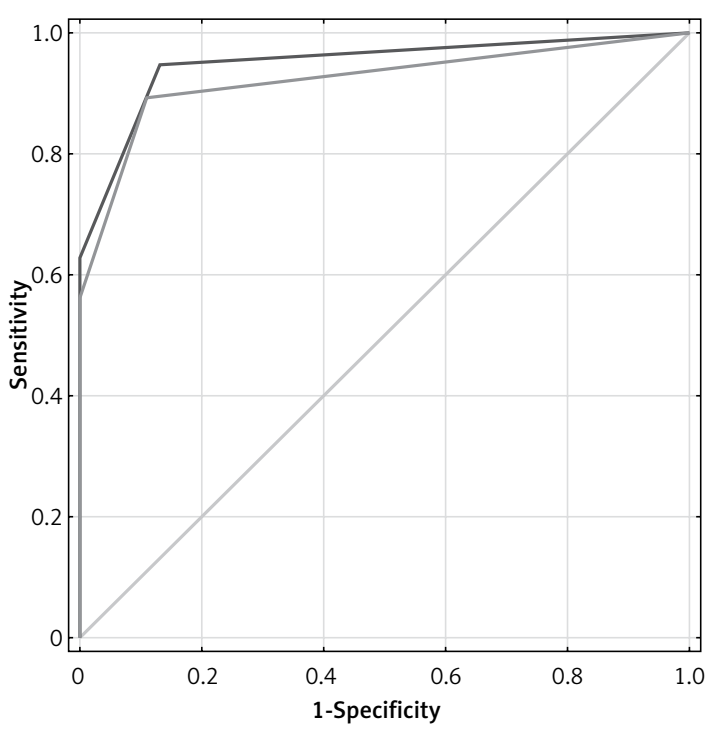

- $\mathrm{T} 2 \mathrm{~A}-\mathrm{T} 2 \mathrm{~B} \quad$ - Reference line

Figure 4. Receiver operating characteristic curves (ROC) during non-nociceptive procedure (T2) for rater $\mathrm{A}(\mathrm{AUC}=0.950)$ and rater $\mathrm{B}(\mathrm{AUC}=0.926)$

in Poland. This is one of the steps in improving quality of care and patient outcome, as defined by international guidelines [2-4]. Clinical observation and the results of prospective clinical trials show that the incidence of pain in critically ill, intubated, mechanically ventilated patients is underreported and untreated. Part of the problem lies in the fact that behavioural pain assessment tools were originally developed and available only in English or French. Because a behavioural pain scale was unavailable for critical care patients in Poland we undertook a process of translation of the CPOT and BPS into Polish [7]. Afterwards we performed this observational study to validate the Polish version of the CPOT to make it available to Polish-speaking populations world-wide. The results of this study add the Polish version to the recently translated and validated Danish and Chinese CPOT scales [8-10].

Our study included unsedated patients who received no hypnotic, only analgesia, namely morphine or fentanyl as an intravenous infusion, to

Table VII. Comparison of Critical Care Pain Observation Tool (CPOT) values between time points for raters A and B

\begin{tabular}{|lccc|}
\hline Assessment & Time & CPOT rater A & CPOT rater B \\
\hline Before NNP-NNP & T1 to T2 & 1.4 & 0.97 \\
\hline NNP - After NNP & T2 to T3 & $2.29^{*}$ & 1.47 \\
\hline Before NP-NP & T4 to T5 & $7.06^{*}$ & $7.00^{*}$ \\
\hline NP-After NP & T5 to T6 & $7.00^{*}$ & $6.90^{*}$ \\
\hline NNP-NP & T2 to T5 & $7.11^{*}$ & $7.06^{*}$ \\
\hline
\end{tabular}

Results of a Wilcoxon signed-rank test. ${ }^{*} p<0.001$. NNP-non-nociceptive procedure, NP-nociceptive procedure. 


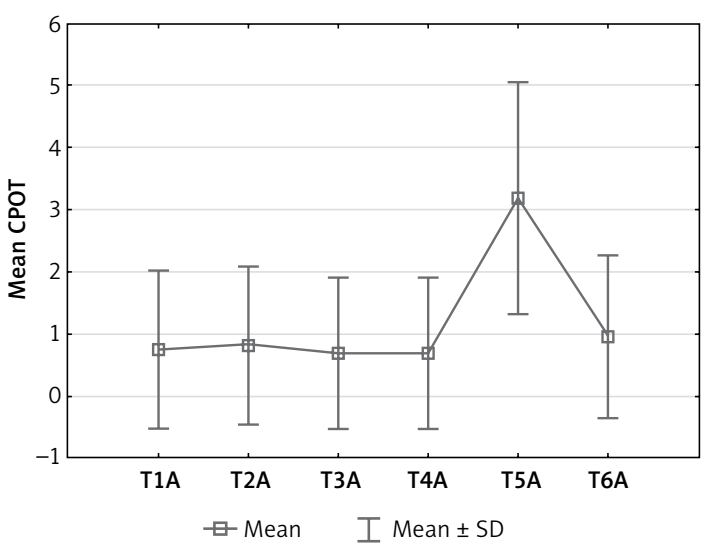

Figure 6. Mean CPOT values $( \pm \mathrm{SD})$ reported by rater $A$ at time points T1-T6; CPOT values at T5 were significantly higher that at other time points; $p<0.001$

tolerate intubation and mechanical ventilation. This approach to ICU treatment is quite unique as it allows the patients to be awake and cooperative. Therefore the majority of patients (58/71, $81.7 \%$ ) were able to self-report pain using the NRS scale. Opioid delivery in this study differs from that recommended by the guidelines, i.e. intermittent boluses - as it was an observational study we did not want to change current practice on our ICU. Continuous opioid infusion is used in our unit as it is a good balance between patient comfort and the available resources, i.e. a limited nurse-topatient ratio.

The Polish version of the CPOT showed very good inter-rater reliability, with ICC above 0.9 for all time points. This is especially important as the CPOT was performed by 9 different observers rater $A$ was the primary investigator and rater $B$ was one out of 8 persons from the study team. We set this goal in contrast to other researchers who only used two observers as CPOT raters at all assessments and reported it as a major limitation to their study [8]. Also Gelinas et al. in the original CPOT validation study, where only two raters col-

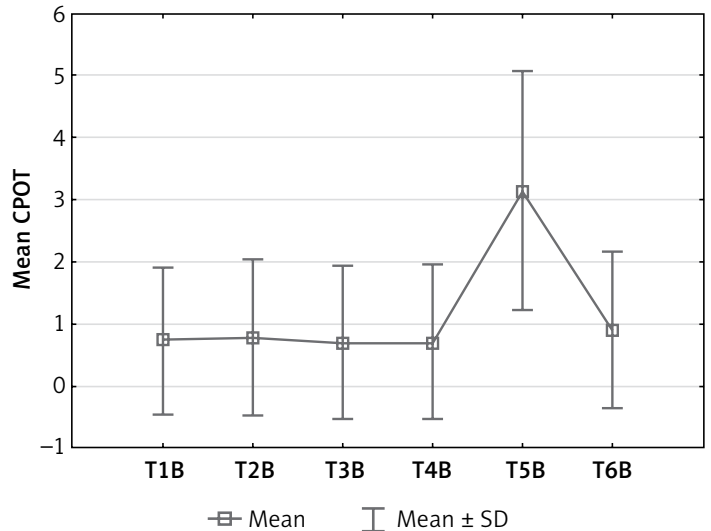

Figure 7. Mean CPOT values $( \pm S D)$ reported by rater $B$ at time points T1-T6; CPOT values at T5 were significantly higher that at other time points; $p<0.001$

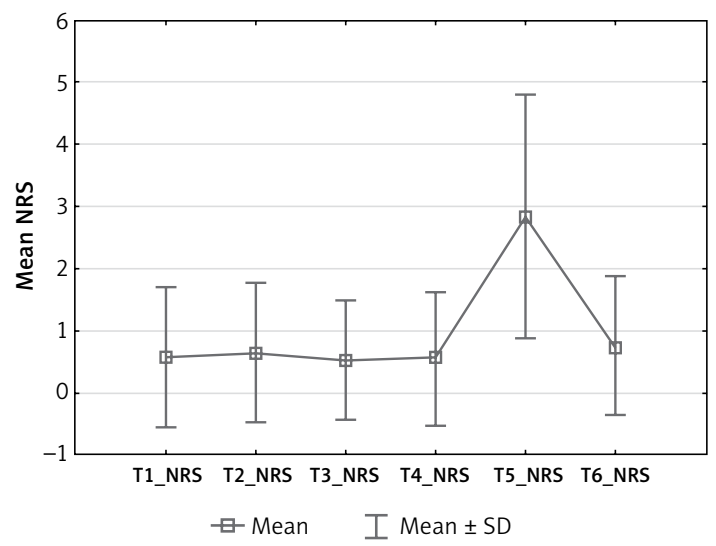

Figure 8. Mean NRS values $( \pm S D$ ) reported by the patient at time points T1-T6; CPOT values at T5 were significantly higher that at other time points; $p<0.001$

lected the data, underlined the importance of using more raters in the evaluation of inter-rater reliability [5]. Therefore it is especially important in the POL-CPOT study that participation of multiple assessors did not lower the inter-rater reliability scores. It must be underlined that pain monitoring

Table VIII. Spearman rank correlation coefficients for correlations between Critical Care Pain Observation Tool (CPOT) and physiological indicators values for 2 raters at 3 time points

\begin{tabular}{|c|c|c|c|c|c|}
\hline Assessment & Time & Heart rate & Systolic BP & Diastolic BP & Mean BP \\
\hline \multicolumn{6}{|l|}{ Rater A: } \\
\hline Before NNP & $\mathrm{T} 1$ & 0.18 & -0.02 & $-0.27^{\star}$ & -0.18 \\
\hline NNP & $\mathrm{T} 2$ & 0.19 & -0.10 & $-0.32^{*}$ & $-0.24^{\star}$ \\
\hline NP & T5 & 0.23 & -0.10 & $-0.28^{*}$ & -0.20 \\
\hline \multicolumn{6}{|l|}{ Rater B: } \\
\hline Before NNP & $\mathrm{T} 1$ & 0.22 & -0.01 & $-0.27^{\star}$ & -0.17 \\
\hline NNP & $\mathrm{T} 2$ & 0.16 & -0.09 & $-0.30^{\star}$ & -0.23 \\
\hline NP & T5 & 0.18 & -0.08 & $-0.28^{*}$ & -0.19 \\
\hline
\end{tabular}

${ }^{\star} p<0.05 . B P-$ blood pressure, NNP-non-nociceptive procedure, NP-nociceptive procedure. 
is traditionally the weakest part of the PAD or DAS Guidelines regarding the available data on the tools. Therefore our study showed that this subjective element of suffering had good resultant ICC coefficients.

Strong correlations ( $R s>0.85$ ) between patients' self-report of pain intensity at rest measured by the NRS and the CPOT score showed criterion validity $(p<0.0001)$. This makes it a reliable tool, especially in the context of other validation studies [8, 11-13]. The original results reported by Gélinas et al. showed that the CPOT has good internal consistency determined by standardized Cronbach's $\alpha(\alpha=0.89)$ with good agreement percentages (80\%) and acceptable inter-rater reliability $(\kappa=0.52-1$; ICC $=0.80-0.93)[14,15]$. On the other hand, the study by Nürnberg et al. validating the Swedish version of the CPOT showed poor inter-rater reliability with a low-weighted $\kappa$ coefficient $(\kappa=0.26)$ [12].

Discriminant validation was determined by higher CPOT scores during painful procedures as compared with non-painful procedures or assessment at rest $(p<0.0001)$. This is consistent with other CPOT validation studies reporting significantly higher scores during nociceptive procedures than at rest or during non-nociceptive procedures $[12,16,17]$. One of the steps was to determine the cut-off values for the Polish version of the CPOT, which was based on a comparison of patients' self-report of presence of pain with the CPOT. Our study shows that the optimal cut-off point for POL-CPOT to detect pain at the nociceptive procedure is $\geq 2$. According to Gelinas and Johnson, the cut-off point for the CPOT lies between 2 and 3 points, where a score of 2 is equivalent to pain [14]. The Danish CPOT validation study showed that cut-off values $\geq 3$ and $\geq 4$ for all included patients were equally good [8]. Multiple other studies have shown optimal cut-off values at $>2[15,18]$. In many countries translations of the CPOT were performed to aid ICU clinicians and validation studies showed its good consistency and validation [8-10, 19, 20].

It is also worth underlining that country-specific differences in pain detection and treatment exist. Interestingly, research shows that nationality-specific differences exist in terms of pain experience. Golicki and Niewada reported that Polish patients present with generally poorer health, especially when regarding the perception of pain [21]. Moreover, the study performed by Mędrzycka-Dąbrowska et al. in 2016 showed that pain management in Poland is still sub-optimal, especially in the elderly population [22]. Therefore adding a validated tool for pain assessment available in the Polish language seems valuable for healthcare professionals in Poland.
Study limitations must also be addressed. We did not assess the Cronbach coefficient as each subquestion in the CРOT scale was validated in previous studies $[5,11,23]$. We collected and analysed the data regarding vital signs both during the nociceptive and non-nociceptive procedures (NP and NNP), although many studies have shown that neither the heart rate nor blood pressure nor respiratory rate is a reliable index for pain in critically ill patients. It has been reported by other authors that vital signs are unreliable signs of pain and may be related to other conditions. No chronic pain syndrome patients or regular narcotic users were included in this study as these conditions are well-known confounding factors. In these conditions the sensitivity to pain increases and it may lead to abnormally high NRS or CPOT scores; therefore to have a clear picture for tool validation purposes these patients were excluded. It must be admitted however that future studies must report pain assessment also in chronic pain patients as their suffering seems to be pronounced in acute pain situations. It should also be underlined that the population studied by us was mainly composed of adult males. Considering that adolescents, children and females may have different thresholds for recognition of pain, one should bear in mind the value of the Polish CPOT in assessing pain in the other populations which have not been studied here. Further research might be required to validate this tool in other populations.

In conclusion, the findings of this study confirm that the Polish version of the Critical Care Pain Observation Tool can be regarded as a valid instrument for assessing pain among critically ill intubated adult patients. This tool is useful and reliable in critically ill patients with a no-hypnotic, opioid-infusion based analgo-sedation protocol. Polish CPOT scores correlated well with patients' self-reported presence of pain and showed excellent inter-rater reliability. This makes the Polish version of the CPOT a reliable pain assessment tool.

\section{Conflict of interest}

The authors declare no conflict of interest.

\section{References}

1. Chanques G, Sebbane M, Barbotte E, Viel E, Eledjam JJ, Jaber $S$. A prospective study of pain at rest: incidence and characteristics of an unrecognized symptom in surgical and trauma versus medical intensive care unit patients. Anesthesiology 2007; 107: 858-60.

2. Barr J, Fraser GL, Puntillo K, et al. Clinical practice guidelines for the management of pain, agitation, and delirium in adult patients in the intensive care unit. Crit Care Med 2013; 41: 263-306.

3. DAS-Taskforce 2015, Baron R, Binder A, Biniek R, Braune $S$, Buerkle $H$. Evidence and consensus based guide- 
line for the management of delirium, analgesia, and sedation in intensive care medicine. Revision 2015 (DAS-Guideline 2015) - short version. Ger Med Sci 2015; 13: Doc19. doi: 10.3205/000223.

4. Vincent JL, Shehabi Y, Walsh TS, et al. Comfort and patient-centred care without excessive sedation: the eCASH concept. Intensive Care Med 2016; 42: 962-71.

5. Gelinas C, Fillion L, Puntillo KA, et al. Validation of the critical-care pain observation tool in adult patients. Am J Crit Care 2006; 15: 420-7.

6. Payen JF, Bru O, Bosson JL, et al. Assessing pain in critically ill sedated patients by using a behavioral pain scale. Crit Care Med 2001; 29: 2258-63.

7. Kotfis K, Zegan-Barańska M, Szydłowski Ł, Żukowski M, Ely WE. Methods of pain assessment in adult intensive care unit patients - Polish version of the CPOT (Critical Care Pain Observation Tool) and BPS (Behavioral Pain Scale). Anaesthesiol Intensive Ther 2017; 49: 66-72.

8. Frandsen JB, O’Reilly Poulsen KS, Laerkner E, Stroem T. Validation of the Danish version of the Critical Care Pain Observation Tool. Acta Anaesthesiol Scand 2016; 60: 1314-22.

9. Li Q, Wan X, Gu C, et al. Pain assessment using the critical-care pain observation tool in Chinese critically ill ventilated adults. J Pain Symptom Manage 2014; 48: 975-82.

10. Hsiung NH, Yang Y, Lee MS, Dalal K, Smith GD. Translation, adaptation, and validation of the behavioral pain scale and the critical-care pain observational tools in Taiwan. J Pain Res 2016; 9: 661-9.

11. Gélinas C, Puntillo KA, Joffe AM, Barr J. A validated approach to evaluating psychometric properties of pain assessment tools for use in nonverbal critically ill adults. Semin Respir Crit Care Med 2013; 34: 153-68.

12. Nürnberg Damström D, Saboonchi F, Sackey PV, Björling G. A preliminary validation of the Swedish version of the critical-care pain observation tool in adults. Acta Anaesthesiol Scand 2011; 55: 379-86.

13. Rijkenberg S, Stilma W, Endeman H, Bosman RJ. Oudemans-van Straaten HM. Pain measurement in mechanically ventilated critically ill patients: behavioral Pain Scale versus Critical-Care Pain Observation Tool. J Crit Care 2015; 30: 167-72.

14. Gélinas C, Johnston C. Pain assessment in the critically ill ventilated adult: validation of the critical-care pain observation tool and physiologic indicators. Clin J Pain 2007: 23: 497-505.

15. Gelinas C, Fillion L, Puntillo KA. Item selection and content validity of the Critical-Care Pain Observation Tool for non-verbal adults. J Adv Nurs 2009; 65: 203-16.

16. Boitor M, Fiola JL, Gelinas C. Validation of the Critical-Care Pain Observation Tool and vital signs in relation to the sensory and affective components of pain during mediastinal tube removal in postoperative cardiac surgery intensive care unit adults. J Cardiovasc Nurs 2016; 31: 425-32.

17. Vazquez M, Pardavila MI, Lucia M, Aguado Y, Margall MA, Asiain MC. Pain assessment in turning proce dures for patients with invasive mechanical ventilation. Nurs Crit Care 2011; 16: 178-85.

18. Gelinas C, Harel F, Fillion L, Puntillo KA, Johnston CC. Sensitivity and specificity of the critical-care pain observation tool for the detection of pain in intubated adults after cardiac surgery. J Pain Symptom Manage 2009; 37 : 58-67.

19. Kwak EM, Oh H. Validation of a Korean translated version of the critical care pain observation tool (CPOT) for
ICU patients [Korean with English abstract]. J Korean Acad Nurs 2012; 42: 76-84.

20. Juarez P, Bach A, Baker M, et al. Comparison of two pain scales for the assessment of pain in the ventilated adult patient. Dimens Crit Care Nurs 2010; 29: 307-15.

21. Golicki D, Niewada M. EQ-5D-5L Polish population norms. Arch Med Sci 2017; 13: 191-200.

22. Mędrzycka-Dąbrowska W, Dąbrowski S, Basiński A, Pilch D. Perception of barriers to postoperative pain management in elderly patients in Polish hospitals with and without a "Hospital Without Pain" Certificate a multi-center study. Arch Med Sci 2016; 12: 808-18.

23. Chanques G, Pohlman A, Kress JP, et al. Psychometric comparison of three behaveoural scales for the assessment of pain in critically ill patients unable to self-report. Crit Care 2014; 18: R160. 